\title{
The complete chloroplast genome sequence of Pseudoroegneria libanotica, genomic features, and phylogenetic relationship with Triticeae species
}

\author{
D.D. WU ${ }^{1,2}$, L.N. SHA ${ }^{1}$, C. TANG ${ }^{1}$, X. FAN ${ }^{1}$, Y. WANG ${ }^{1}$, H.Y. KANG ${ }^{1}$, H.Q. ZHANG ${ }^{1}$, \\ and Y.H. ZHOU ${ }^{1,2 *}$ \\ Triticeae Research Institute, Sichuan Agricultural University, Wenjiang 611130, Sichuan, P.R. China ${ }^{1}$ \\ Key Laboratory of Crop Genetic Resources and Improvement, Ministry of Education, \\ Sichuan Agricultural University, Wenjiang 611130, Sichuan, P.R.China ${ }^{2}$
}

\begin{abstract}
Pseudoroegneria libanotica is an important herbage diploid species possessing the St genome. The St genome participates in the formation of nine perennial genera in Triticeae (Poaceae). The whole chloroplast (cp) genome of $P$. libanotica is $135026 \mathrm{bp}$ in length. The typical quadripartite structure consists of one large single copy of $80634 \mathrm{bp}$, one small single copy of $12766 \mathrm{bp}$ and a pair of inverted regions (20 $813 \mathrm{bp}$ each). The cp genome contains 76 coding genes, four ribosomal RNA and 30 transfer RNA genes. Comparative sequence analysis suggested that: 1) the $737 \mathrm{bp}$ deletion in the cp of P. libanotica was specific in Triticeae species and might transfer into its nuclear genome; 2) hotspot regions, indels in intergenic regions and protein coding sequences mainly led to the length variation in Triticeae; 3) highly divergence regions combined with negative selection in $r p l 2, r p s 12, \operatorname{css} A, r p s 8, n d h H, p e t D, n d h K, p s b M$, rps3, rps 18 , and $n d h A$ were identified as effective molecular markers and could be considered in future phylogenetic studies of Triticeae species; and 4) ycf3 gene with rich cpSSRs was suitable for phylogeny analysis or could be used for DNA barcoding at low taxonomic levels. The cpSSRs distribution in the coding regions of diploid Triticeae species was shown for the first time and provided a valuable source for developing primers to study specific simple sequence repeat loci.
\end{abstract}

Additional key words: hot-spot regions, phylogenetic tree, sequence transfer, simple sequence repeats.

\section{Introduction}

Chloroplasts are multifunctional organelles possessing own genetic material. The function of chloroplasts is to perform photosynthesis, to storage starch, to metabolize nitrogen and sulfate, and synthesize chlorophyll, carotenoids, fatty acids, and nucleic acids (Neuhaus and Emes 2000, Rodríguez-Ezpeleta et al. 2005). Chloroplast (cp) genome consists of two copies of inverted repeats (IRs) separating the large single-copy region (LSC, 80 -
$90 \mathrm{~kb}$ ) and small single-copy region (SSC, $16-27 \mathrm{~kb}$ ) (Palmer 1991). The gene content and structure of the majority of angiosperm cp genomes are highly conserved (Wicke et al. 2011), but expansion and contraction of the IR, as well as gene inversion, loss and rearrangements, even transferring into nuclear genome have been reported (Matsuo et al. 2005). Because of the highly conservative nature and slow evolutionary rate of the $\mathrm{cp}$ genome,

Submitted 17 December 2016, last revision 13 May 2017, accepted 6 June 2017.

Abbreviations: BS - bootstrap value; CNS - conserved noncoding sequences; cp - chloroplast; cpSSR - chloroplast simple sequence repeat; $\mathrm{d}_{\mathrm{N}} / \mathrm{d}_{\mathrm{S}}$ - ratio of nonsynonymous and synonymous substitution rates; indel - insertion and deletion; IR - inverted region; LSC large single copy; ML - maximum likelihood; rRNA - ribosomal RNA; SSC - small single copy; TE - transposable element; tRNA transfer RNA.

Acknowledgments: The authors are thankful to the National Natural Science Foundation of China (Grant Nos. 31470305, 31270243, and 31200252), the National Key Research and Development of China (2016YFD0102000), and the fund from the Science and Technology Bureau and Education Bureau of Sichuan Province, China. We are very grateful to the American National Plant Germplasm System (Pullman, Washington, USA) for providing seed material for this study. The first two authors contributed equally to this work.

* Corresponding author; fax: (+86) 28 82650350, e-mail: zhouyh@sicau.edu.cn 
extensive molecular evolution data demonstrated that cpDNA sequences are suitable and invaluable tool for phylogenetic relationships and the maternal donors studies in polyploids (Mason-Gamer et al. 2002, Zhang et al. 2009, Sha et al. 2010).

The tribe Triticeae, an economically important plant group, includes not only economic crop species (e.g., wheat, rye, triticale, and barley) but also significant forage resources (e.g., Pseudoroegneria strigosa, Elymus sibiricus, Leymus chinensis). Pseudoroegneria is a perennial genus of Triticeae, including 11 species (Yen and Yang 2011). The basic genomic constitution was designed as St genome. Extensive cytological studies have suggested that the St genome with the other basic genomes $\mathrm{W}, \mathrm{H}, \mathrm{Y}, \mathrm{P}$, and $\mathrm{E}$ constitute in various combinations different genera such as Douglasdeweya (StP), Elymus (StH), Roegneria (StY), Trichopyrum (StE), Anthosachne (StYW), Campeiostachys (StYH), Kengyilia (StYP), Psammopyrum (EStP), and Pascopyrum (StHNsXm). The $\mathrm{P}, \mathrm{H}$, and $\mathrm{W}$ genomes are derived from Agropyron, Hordeum, and Australopyrum, respectively (Jensen 1989, Torabinejad and Mueller 1993, Lu 1994, Yen and Yang 2011). Phylogenetic analysis based on limited cpDNA sequences (rpoA, $n d h F$, trnH-psbA, etc.) have concluded that Pseudoroegneria species served as the maternal donor during the

\section{Materials and methods}

Plant material, DNA extraction, and high-throughput sequencing: Genomic DNA was extracted from fresh young leaves of diploid Pseudoroegneria libanotica (Hackel) D.R. Dewey (PI228392) using the mCTAB method (Li et al. 2013a). This material was planted at the Greenhouse of Sichuan Agriculture University in 2015 and the voucher specimen was deposited in the herbarium of the Triticeae Research Institute, Sichuan Agricultural University, China. Genomic DNA was fragmented by ultrasound treatment, and gel extraction was used to obtain DNA fragments with sizes between 400 - 600 bp. The NEBNext ${ }^{\circledR}$ kit (New England Biolabs, Ipswich, MA, USA) was applied to construct a 500 bp DNA library, and Hiseq 4000 PE150 (Illumina, CA, USA) was utilized for sequencing.

Genome assembly, annotation, comparison and sequence analyses: High-throughput sequencing data were assembled by SPAdes 3.6.1 (Bankevich et al. 2012), CLC Genomics Workbench 8 and SOAPdenovo2 (Luo et al. 2012). Chloroplast contigs were screened from whole genome sequences using the BLAST process. Assembly of cp reads was conducted with the help of Sequencher 4.10 (Gene Codes, Ann Arbor, MI, USA). Geneious 8.1 (Kearse et al. 2012) was used to map all reads to the total $\mathrm{cp}$ sequence to confirm the validity of contigs. Specific primers were designed for PCR in order speciation of the St-containing polyploid species in Triticeae (Redinbaugh et al. 2000, Mason-Gamer et al. 2002, Liu et al. 2006, Sha et al. 2010, Dong et al. 2015). Nevertheless, on the basis of $\operatorname{trnL-F}$ region Zhang et al. (2009) demonstrated that the maternal donor of Kengyilia melanthera and $K$. thoroldiana may originate from Agropyron (P genome) species. Although these studies add to our understanding of phylogeny and maternal donor of Triticeae species, discordance still exists in St-containing species due to incomplete and different $\mathrm{cp}$ molecular markers. A robust phylogenetic analysis requires a substantial sequence length and primarily uniparental inheritance, which makes the $\mathrm{cp}$ genome a valuable source for genetic markers (Provan et al. 2001). The complete plastid genome information is very useful within maternal donors and it helps to decipher species evolution (Gornicki et al. 2014, Ma et al. 2014).

In this work, Illumina reads from a partial genome sequence of Pseudoroegneria libanotica were used to assemble the cp genome. The objectives in this study were: 1) to determine the complete $\mathrm{cp}$ genome of $P$. libanotica, and 2) to compare whole $\mathrm{cp}$ genomes among eight diploid Triticeae species. Based on those data sets, we revealed a solid resource for phylogenetic studies and comparative genomics of the Triticeae species.

to repair gaps. PCR condition: at $94{ }^{\circ} \mathrm{C}$ for $4 \mathrm{~min}$, 34 cycles at $94{ }^{\circ} \mathrm{C}$ for $30 \mathrm{~s}$, at $55^{\circ} \mathrm{C}$ for $30 \mathrm{~s}$, at $72{ }^{\circ} \mathrm{C}$ for $1.5 \mathrm{~min}$, then at $72{ }^{\circ} \mathrm{C}$ for $10 \mathrm{~min}$. The PCR products were sequenced by ABI 3730 (Applied Biosystems, Foster City, CA, USA).

The cp genome was annotated using the program DOGMA (Wyman et al. 2004), coupled with manual corrections for start and stop codons. The coding and RNA genes were identified using BLASTX and BLASTN. The border of exons and introns, and some short exons (such as rps16, petB, petD) which could not be identified by $D O G M A$, were referred to published sequences. OrganellarGenomeDRAW (http://ogdraw.mpimp-golm. mpg.de/index.shtml) was used to construct the total $\mathrm{cp}$ genome structure. Adobe Illustrator CS5 was utilized for editing.

Chloroplast simple sequence repeats (cpSSRs) of P. libanotica were screened by SSRHunter1.3 (Li and Wan 2005), with thresholds of eight repeat units for mononucleotide SSRs, four repeat units for di- and trinucleotide SSRs ( 8 and $12 \mathrm{bp}$, respectively), and three repeat units for tetra- and penta-nucleotide SSRs (12 and $15 \mathrm{bp}$, respectively). Eight complete plastid genomes of cereal species were downloaded from NCBI (http://www. ncbi.nlm.nih.gov/) (Table 1 Suppl.). To align the cp genome of $P$. libanotica with other cp genomes $m$ VISTA (Frazer et al. 2004) was employed in Shuffle-LAGAN 
mode. Alignment of the complete cp genome and coding regions of eight diploid Triticeae species were conducted by MAFFT (http://mafft.cbrc.jp/alignment/ server/) independently. Protein coding sequences alignments were carried out by DNAMAN 6.0.3.48 (Lynnon Biosoft, Quebec, Canada). To detect selective constraints on the coding portions (introns were excluded) of all protein coding genes, the ratio of non-synonymous to synonymous substitution $\left(\mathrm{d}_{\mathrm{N}} / \mathrm{d}_{\mathrm{S}}\right)$ were computed using the modified Nei-Gojobori method in MEGA 6.0 software (Tamura et al. 2013). Pairwise sequence divergences were calculated by using the Kimura's two-parameter model and nucleotide polymorphisms estimates for each gene was completed by DNASp 4.0 (Rozas et al. 2003).

Phylogenetic analyses: Phylogenetic analysis was conducted using maximum likelihood (ML) with two sets of the data-complete $\mathrm{cp}$ (non-coding and protein coding) and protein-coding sequence (cds). The evolutionary model used for the phylogenetic analysis was determined using ModelTest v. 3.0 with Akaike Information Criterion (AIC) (Posada and Crandall 1998). The optimal model identified was generalized time-reversible + gamma distribution + proportion of invariable sites $(\mathrm{GTR}+\mathrm{G}+\mathrm{I})$. The ML analysis was performed using PAUP*4.0b10 (Swofford and Sinauer Associates, http://www. sinauer.com). The ML heuristic searches were performed with 100 random addition sequence replications and tree bisection and reconnection (TBR) branch swapping algorithm. The robustness of the trees was estimated by bootstrap support (BS). Trees were viewed in FigTree v. 1.4.1 (http://tree.bio.ed.ac.uk/).

\section{Results}

The total reads obtained from $P$. libanotica by Illumina sequencing were 13265148 and 102083 mapped reads were screened for the entire $\mathrm{cp}$ genome. The whole $\mathrm{cp}$ genome of $P$. libanotica was $135026 \mathrm{bp}$ in length. The typical quadripartite structure consisted of one LSC of $80634 \mathrm{bp}$, one SSC of $12766 \mathrm{bp}$, and a pair of IRs of 20813 bp each (Fig. 1 Suppl.). The overall guanine/cytosine (GC) content of the $P$. libanotica $\mathrm{cp}$ genome was $38.35 \%$. The GC content of the IR regions $(44.02 \%)$ was higher than that of the LSC (36.39\%) and
SSC regions $(32.23 \%)$. High GC content in IR regions is mainly due to the presence of the rRNA gene group.

The cp genome of $P$. libanotica contained 110 encoding genes, including 30 tRNA, four rRNA (23S, 16S, 5S, and 4.5S) and 76 protein coding genes. Thirteen genes included one intron and one gene (ycf3) included two introns. Based on the position of 76 protein coding regions, 60 of them located in LSC, 10 in SSC, and six in IR regions. The $\mathrm{cp}$ genes can be divided into three functional types (Table 1). The first type contains

Table 1. Genes in the chloroplast genome of Triticeae. Intron-containing genes are marked by asterisks.

\begin{tabular}{|c|c|c|}
\hline Category for genes & Group of gene & Name of gene \\
\hline \multirow[t]{8}{*}{ Photosynthesis related genes } & Rubisco & $r b c L$ \\
\hline & photosystem I & $p s a A, B, C, I, J$ \\
\hline & assembly/stability of photosystem I & $* y c f 3, y c f 4$ \\
\hline & photosystem II & $p s b A, B, C, D, E, F, H, I, J, K, L, M, N, T, Z$ \\
\hline & ATP synthase & atp $A, B, E, * F, H, I$ \\
\hline & cytochrome $b / f$ complex & petA, $* B, * D, G, L, N$ \\
\hline & cytochrome $c$ synthesis & $\operatorname{ccs} A$ \\
\hline & NADPH dehydrogenase & ${ }^{*} n d h A,{ }^{*} B, C, D, E, F, G, H, I, J, K$ \\
\hline \multirow{3}{*}{$\begin{array}{l}\text { Transcription and translation } \\
\text { related genes }\end{array}$} & transcription transcription & $\operatorname{rpo} A, B, C 1, C 2$ \\
\hline & ribosomal proteins & $\begin{array}{l}r p s 2,3,4,7,8,11,12,14,15,16,18,19 ; \\
{ }^{*} r p l 2,14, * 16,20,22,23,32,33,36\end{array}$ \\
\hline & translation initiation factor & infA \\
\hline \multirow[t]{2}{*}{ RNA genes } & ribosomal RNA & $r r n 5, r r n 4.5, r r n 16, r r n 23$ \\
\hline & transfer RNA & $\begin{array}{l}* \text { trnA-UGC, C-GCA, D-GUC, E-UUC, F-GAA, } \\
{ }^{*} G-U C C,{ }^{*} G-G C C, H-G U G, I-C A U,{ }^{*} I-G A U, \\
* K-U U U, L-C A A,{ }^{*} L-U A A, L-U A G, f M-C A U I, \\
M-C A U, N-G U U, P-U G G, Q-U U G, R-A C G, \\
R-U C U, S-G C U, S-G G A, S-U G A, T-G G U, \\
T-U G U, V-G A C, * V-U A C, W-C C A, Y-G U A\end{array}$ \\
\hline Other genes & $\begin{array}{l}\text { RNA processing } \\
\text { carbon metabolism } \\
\text { proteolysis }\end{array}$ & $\begin{array}{l}\operatorname{matK} \\
\operatorname{cem} A \\
\operatorname{clp} P\end{array}$ \\
\hline
\end{tabular}


60 genes, which are involved in transcription and translation. The second type contains 47 genes involved in photosynthesis. The third type contains three genes, responsible for the biosynthesis of amino acids and fatty acids and of unknown function.

Chloroplast SSRs are generally short mononucleotide tandem repeats and randomly spread throughout $\mathrm{cp}$ genomes, stretching from one to six nucleotide units. Perfect SSRs which are longer than $8 \mathrm{bp}$ were detected in the $P$. libanotica $\mathrm{cp}$ genome. This threshold was set because SSRs of $8 \mathrm{bp}$ or longer are prone to slipstrand mispairing, which is thought to be the primary mutational mechanism causing their high level of polymorphism (Rose and Falush 1998, Huotari and Korpelainen 2012). In $P$. libanotica complete cp genome, $128 \mathrm{cpSSRs}$ repeats were found. Total cpSSRs consisted of five types: mononucleotides (91 SSRs: $71 \%$ ), dinucleotides (28 SSRs: $22 \%$ ), trinucleotides (two SSRs: $1 \%$ ), tetranucleotides (six SSRs: $5 \%$ ), and pentanucleotides (only one SSR: $1 \%$ ). There were 98 (76.6\%), $15(11.7 \%)$, and $15(11.7 \%)$ SSR repeats distributing in LSC, IR, and SSC regions, respectively (Fig. 1A). There were $38(29.7 \%)$ SSRs in exon regions, $11(8.6 \%)$ in intron regions, and $79(61.7 \%)$ in intergenic spacer (Fig. $1 B$ ). According to the distribution of SSR loci in coding genes of $\mathrm{cp}$ genome, the coding region of $r p o C 2$ contained the highest number of repeats with 6 SSRs rich in adenosine/thymine $(\mathrm{A} / \mathrm{T})$. Coding regions of $n d h F$ and $y c f 3$ included five SSRs each (Table 2 Suppl.).

Structural mutations occur at hot-spot regions,

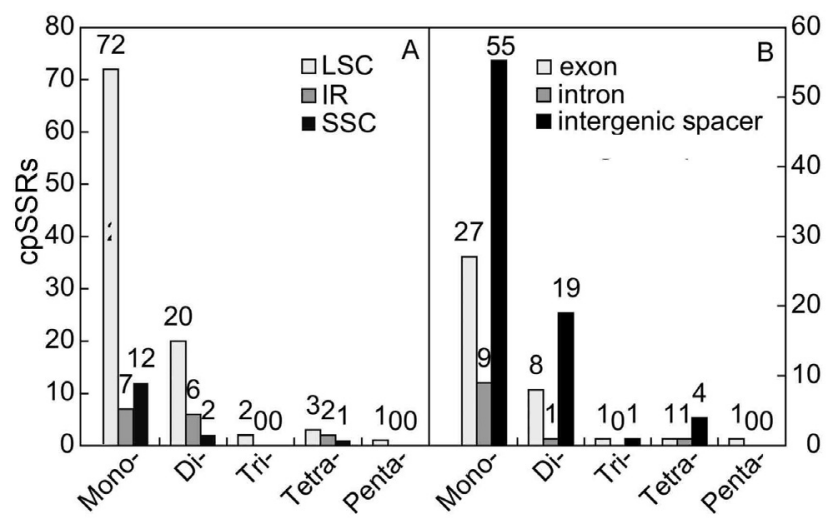

Fig. 1. The distribution of cp simple sequence repeats (cpSSRs) in Pseudoroegneria libanotica. A - SSR location according to type: mononucleotide, dinucleotide, trinucleotide, tetranucleotide, and pentanucleotide repeats. The number of SSR type in LSC, IR and SSC are displayed. $B$ - Different SSRs type distribute among exon, intron, and intergenic spacer.

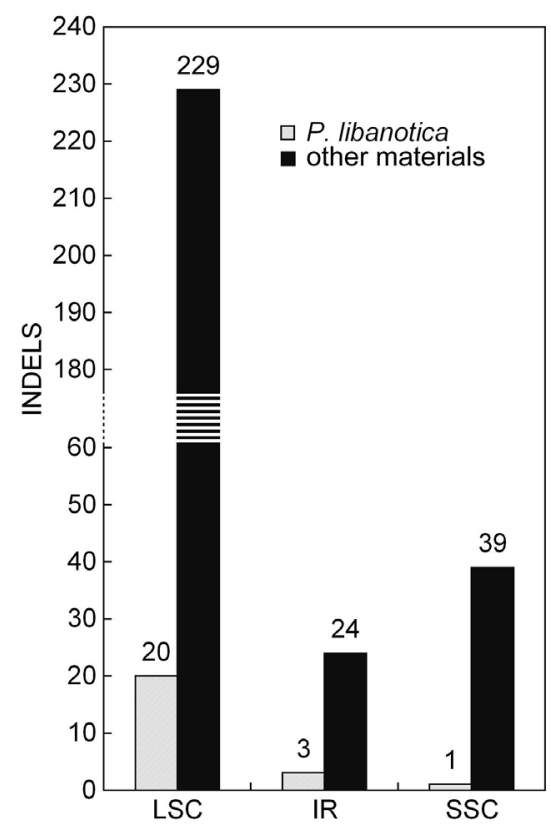

Fig. 2. Insertions and deletions in Pseudoroegneria libanotica and other species. containing AT-rich content which contribute to the variation of genome size, rather than randomly. The cpDNA is well-known by AT-rich. Gene spacer regions are especially AT-rich. The overall AT content in the cp sequences of Triticeae diploid species is not as high as in liverwort (61.65\% and $80 \%$, respectively) whereas the intergenic regions of rps4-trnL-trnL (about $1600 \mathrm{bp}$ ) and rbcL-psaI (about 1200 bp) in Triticeae diploid species showed an AT content of more than $80 \%$ (Fig. 2 Suppl.). $\operatorname{Tr} n L-F$, a widely used phylogenetic analyses marker, belongs to rps4-trnL-trnL hot-spot region. The hot-spots region $r b c L$-psaI includes the $r p l 23$ gene, which exhibits multiple mutations in different Triticeae diploid species. In the LSC region of $P$. libanotica, rpl23 fulfils its function normally while it turned out to be a pseudogene or completely lost in the same position of other Triticeae diploid species.

Comparison of seven Triticeae cp genomes except the outgroup Bromus vulgaris (Table 1 Suppl.), revealed that Triticum monococcum (136886 bp) and T. urartu (136 $865 \mathrm{bp}$ ) possessed the biggest cp genome. Hordeum jubatum (136826 bp) and $H$. vulgare subsp. vulgare (136 462 bp) were second in size. Aegilops speltoides var. ligustica (135 660 bp), Ae. tauschii (135 568 bp), and 
Secale cereale (135 $604 \mathrm{bp}$ ) had a cp genome of similar size. P. libanotica (135026 bp) exhibited the smallest $\mathrm{cp}$ genome. $P$. libanotica displayed the highest GC content (38.35\%), followed by Ae. speltoides var. ligustica (38.31\%) and Ae. tauschii (38.34\%). The lowest GC content showed T. monococcum $(38.27 \%$ ) and T. urartu (38.2 8\%). Moreover, the total number of $\mathrm{cp}$ genes differed among the Triticeae species. Most of Triticeae species contained about 76 coding genes. $H$. vulgare subsp. vulgare had 75 coding regions in all and lacked $y c f 4, S$. cereale encoded 75 genes. In $S$. cereale, 73 of the protein coding regions were the same as in other species while it was short for rpl20 and rpl23. However, $S$. cereale possessed two unique $y c f$ regions ( $y c f 2$ and $y c f 15)$. Four conserved rRNAs were identified in all diploid Triticeae species.

The organization of the $\mathrm{cp}$ genome is rather conserved within diploid Triticeae species (Fig. 3 Suppl.) but 316 indels were found in total and 250,27 , and 40 indels in the LSC, IR and SSC regions, respectively (Fig. 2). Most of indels were located in intergenic spacer. In contrast, six indels exist in protein coding regions. In $A e$. speltoides var. ligustica and Ae. tauschii, the rpl2 gene lost a large proportion of the intron (total intron is $651 \mathrm{bp}$, lost $586 \mathrm{bp}$ ) and the complete exon-2 with respect to $P$. libanotica (Table 3 Suppl.). Similarly, petB, petD, rpl16, and rps12 (in IRa) genes lost their introns in Ae. speltoides var. ligustica, Ae. tauschii, S. cereale, T. monococcum, and T. urartu, respectively, whereas they retained in $P$. libanotica. The infA gene length in $P$. libanotica (342 bp) was larger than in Ae. speltoides var. ligustica, Ae. tauschii, and $S$. cereale. The $P$. libanotica retained one copy of rpl23 in LSC. Nevertheless, it showed early termination and formed a pseudo-gene in Ae. speltoides var. ligustica. The rpl23 is absent in the LSC region of $H$. vulgare subsp. vulgare, Ae. tauschii, and H. jubatum while it was completely lost in cp genome of $S$. cereale.

$P$. libanotica contains 20 indels, 16 of them situated in LSC, three in IR, and one in SSC region (Fig. 2). Most of those indels were less than $100 \mathrm{bp}$ in length. The longest sequences change was $737 \mathrm{bp}$ absences in the trnI-trnL intergenic spacer of IRa and IRb regions in spite of IR regions were considered as more stable than single copy regions (LSC and SSC).

The non-synonymous $\left(d_{N}\right)$ and synonymous $\left(d_{S}\right)$
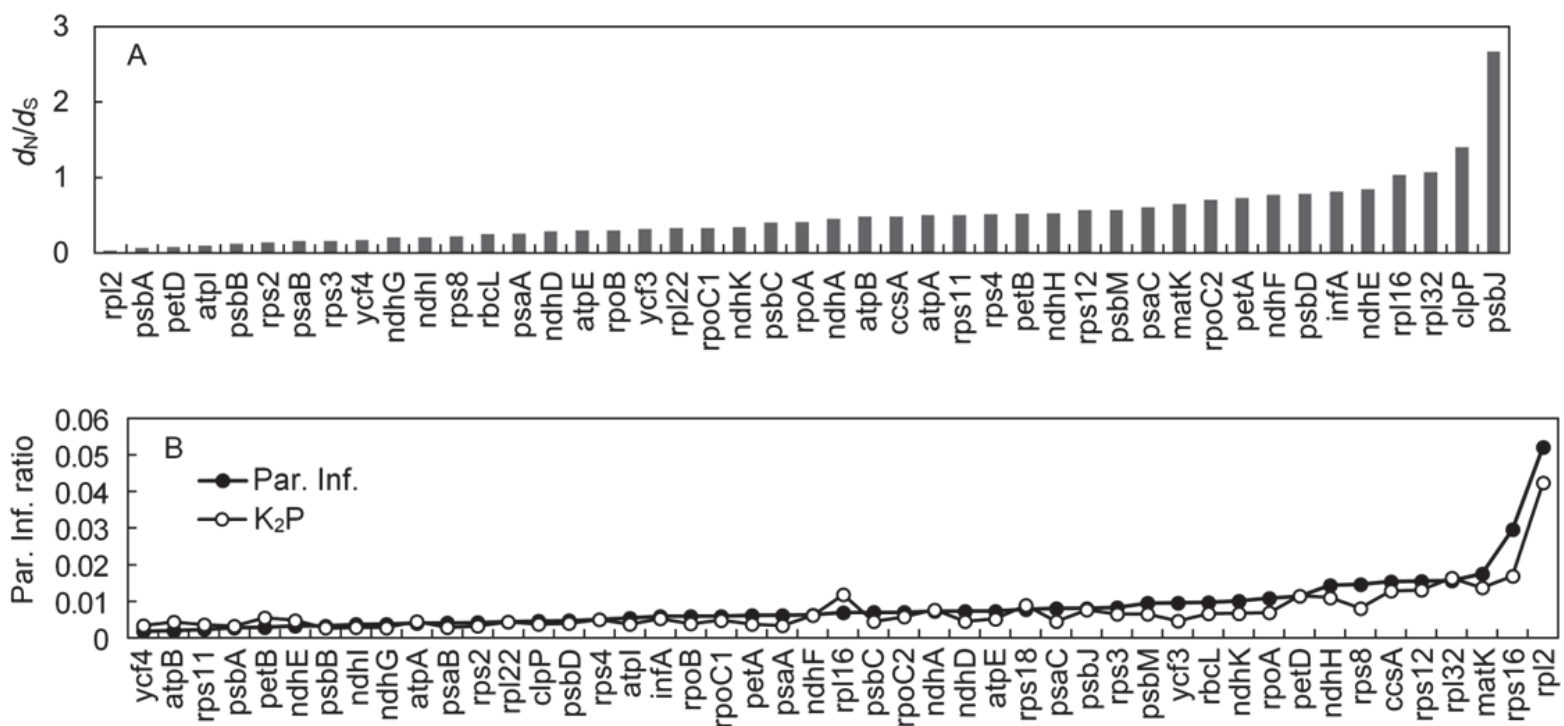

Fig. 3. Selective pressures in protein coding genes of eight diploid Triticeae species. $A-\mathrm{d}_{\mathrm{N}} / \mathrm{d}_{\mathrm{S}}$ in protein coding genes in eight diploid Triticeae species (excluding genes where $\mathrm{d}_{\mathrm{N}}$ and/or $\mathrm{d}_{\mathrm{S}}=0$ ). $B$ - Evolutionary rate of chloroplast genes in eight diploid Triticeae species (excluding genes where $\mathrm{d}_{\mathrm{N}}$ and/or $\mathrm{d}_{\mathrm{S}}=0$ ). Par. Inf. - parsimony-informative, $\mathrm{K} 2 \mathrm{P}=$ Kimura's two-parameter model.

substitution rates $\left(\omega=\mathrm{d}_{\mathrm{N}} / \mathrm{d}_{\mathrm{S}}\right)$ of $>1,=1$ and, $<1$ indicate positive selection, neutral evolution and purifying selection on the coding portions, respectively (Yang and Bielawski 2000). Parsimony-informative (Par. Inf.) ratios and evolutionary rate of coding regions were intended to analyse the most divergent coding regions in our species.

No changes were observed in the synonymous rate of psaI, rpl33, rpl36 and the nonsynonymous rate of the $a t p F, \operatorname{cem} A, n d h B$ (IR region), $n d h C, n d h J$, petL, psbE, psbH, psbI, psbK, psbL, rpl14, rpl20, rps14, rps15 (IR region) and rps19 (IR region). Furthermore, atpH, pet $G$, pet $N, p s b F, p s b N, p s b T, r p l 23$ (IR region), rps7 (IR region) and rps 12 (IR region) showed no synonymous and non-synonymous changes. Most of the genes in IR ( $n d h B, r p s 15, r p s 19, r p l 23, r p s 7$ and rps12) had no changes in the synonymous rate and/or nonsynonymous rate. The $\mathrm{d}_{\mathrm{N}} / \mathrm{d}_{\mathrm{S}}$ ratio indicates most of the protein coding sequences got through purifying selection, except four genes (clpP, psbJ, rpll6, and rpl32) underwent positive selection (Fig. $3 A$ ). 
Parsimony-informative characters and pairwise sequence divergences in coding regions showed similar changing tendency (Fig. 3B). Most cp genes contained less mutation. The rpl2, located in IRs, possessed the highest percentage of Par. Inf. characters and evolutionary rate $(0.0521$ and 0.0424 , respectively), followed by rps16 (0.0296 Par. Inf. and 0.0169 pairwise sequence divergences). In addition, analysis of $\operatorname{mat} K, \operatorname{rps} 12, \operatorname{css} A$, $r p s 8, n d h H$, petD, $n d h K, r p o A, r b c L, p s b M, n d h A, r p s 18$, rps3, and $n d h F$ coding regions analysis showed a high Par. Inf. ratio (ranging from 0.0175 to 0.0063 ) and evolutionary rate $(0.0164$ to 0.0061$)$.

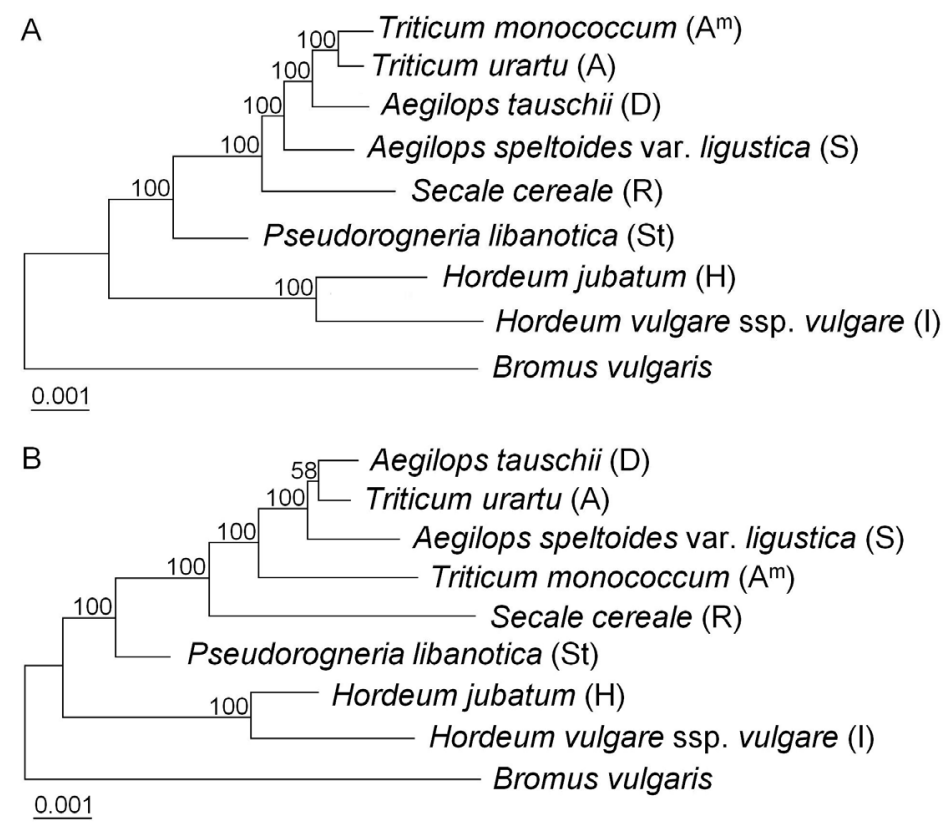

Fig. 4. Phylogenetic analyses based on complete cp sequences and coding regions, using the maximum likelihood (ML) tree. Numbers at nodes indicate bootstrap values (BS). $A$ - ML tree based on the whole cp genomes; $B$ - ML tree based on the coding region of cp genomes.

Bromus vulgaris was used as outgroup. The ML analysis of whole $\mathrm{cp}$ sequences yielded a single phylogenetic tree (-Ln likelihood $=220179.81568)$, with the assumed nucleotide frequencies $\mathrm{A}=0.31140$, $\mathrm{C}=0.18900, \mathrm{G}=0.18980, \mathrm{~T}=0.30980, \alpha$ shape parameter $=0.7954$, and the proportion of invariable sites $=0.778$. Numbers above ML tree branches are bootstrap support (BS). The phylogenetic tree showed that Hordeum was firstly separated from the other Triticeae diploid species. $P$. libanotica had a relatively far relationship with Triticum. S. cereale was aparted from Triticum/Aegilops complex clade gradually with high statistical support. Based on the whole cp genome phylogenetic analysis, Ae. speltoides var. ligustica (S) separated first, followed by Ae. tauschii (D), T. monococcum $\left(\mathrm{A}^{\mathrm{m}}\right)$, and T. urartu (A) with $100 \%$ BS in Triticum/Aegilops complex (Fig. 4A).

The ML analysis of coding region sequences resulted in a single phylogenetic tree (-Ln likelihood =

\section{Discussion}

Cp genomic features of $\boldsymbol{P}$. libanotica: Previous studies have shown an association between repeats and genome
93615.16235), with the assumed nucleotide frequencies $\mathrm{A}=0.29990, \mathrm{C}=0.18110, \mathrm{G}=0.20810, \mathrm{~T}=0.31090$, $\alpha$ shape parameter $=0.8795$, and the proportion of invariable sites $=0.7704$. Hordeum indicated more far relationship with other diploid species and was followed by $P$. libanotica. $S$. cereale formed a group with Triticum/Aegilops complex. But the genetic differentiation of Triticum/Aegilops complex was more complex. In Triticum/Aegilpos complex sub-clade, T. urartu (A), Ae. tauschii (D), and Ae. speltoides var. ligustic $(\mathrm{S})$ clustered together with low support rate and deviated from $T$. monococcum $\left(\mathrm{A}^{\mathrm{m}}\right)$ based on the protein coding regions genetic analysis (Fig. 4B).

Phylogenetic analyses based on $\mathrm{cp}$ protein coding sequences yielded no well-supported contradicting topologies. In contrast, complete cp sequences resulted in quite high node support values $(\mathrm{BS}=100 \%)$ and identical topologies.

rearrangement breakpoints (Lee et al. 2007, Guisinger et al. 2011). The cpSSRs and indels were mainly 
distributed in the LSC region of $P$. libanotica. This observation could be explained by the large genome size, low GC content, and higher point mutation rate in comparison to the IR region. LSC occupies $59.72 \%$ of the complete $\mathrm{cp}$ genome of $P$. libanotica. The GC content of single copy regions (36.39 and $32.23 \%$ in LSC and SSC regions, respectively) were less than in IR regions (44.02\%). Li et al. (2013b) observed that IR regions of cp genome were more conserved in land plants compared to the single copy regions. Perry and Wolfe (2002) showed that the nucleotide substitution rate is 2.3 times higher in the single-copy regions relative to the inverted repeats. The cpSSRs and indels mainly distributed in the non-coding regions of the cp genome, indicating an uneven distribution of SSRs and indels within the $\mathrm{cp}$ genomes. The distribution preference of cpSSRs and indels in $P$. libanotica is in accordance with previous studies in cultivated olives and Salvia miltiorrhiza (Mariotti et al. 2010, Qian et al. 2013).

It is worth pointing out that a $737 \mathrm{bp}$ deletion was located in the trnI-trnL intergenic spacer of IRa and IRb region in $P$. libanotica. Interspecies differentiation, transposable elements (TE), or gene transfer might explain this deletion. We preferred the possibility of transfer of the $737 \mathrm{bp}$ sequence from the $\mathrm{cp}$ into its nuclear genome. The hypothesis of interspecific differentiation was rather unlike because the $737 \mathrm{bp}$ absence existed in all $\mathrm{cp}$ genomes of Triticeae species. The specific deletion even showed high similarity (identify $=98 \%$ ) with Pooideae species. Consequently, the 737 bp deletion might be a relatively conserved sequence existing in all of Pooideae. The TEs were out of the question because of the $737 \mathrm{bp}$ failed to match with any $\mathrm{TE}$ in the Triticeae repeat (http://wheat. pw.usda.gov/ITMI/repeats/). Following evidences support our hypothesis that the loss of the $737 \mathrm{bp}$ fragment was caused by gene transfer. Primarily, numerous researches demonstrated that the nuclear genomes of higher plants harbour a significant amount of promiscuous DNA of plastid origin (Ayliffe et al. 1998, Timmis et al. 2004). Moreover, high sequence similarity exists in the horizontal transfer from different host species (Schaack et al. 2010). BLAST analysis was carried out and we found that the $737 \mathrm{bp}$ deletion was exactly similar (identities $=99 \%$ ) to a genomic scaffold of chromosome 3B of T. aestivum and it possess high similarity (identify $=98 \%$ ) with $\mathrm{cp}$ sequences in Pooideae species.

Variation and phylogenetic relationship within diploid Triticeae species: Generally, the organization of the cp genome is relatively conserved within Triticeae, whereas length variation is caused by the presence of some hotspot regions and sequences losses. The whole cp genome size of eight Triticeae species varied from $135026 \mathrm{bp}$ (P. libanotica) to $136886 \mathrm{bp}$ (T. monococcum). On one hand, the genome size varies along with the changes of the AT-rich content in hot-spot regions (Kim and Lee 2004). TrnL-F sequence, a part of an AT-rich region in rps4-trnL-trnL, has been extensively applied to evolutionary investigation in Triticeae owing to its abundant informative-sites (Zhang et al. 2009, Dong et al. 2015). Various rpl23 genes in rbcL-psal hot-spot region are presumed to be translocated by illegitimate recombination mediated by these short repeat sequences in diploid Triticeae species (Ogihara et al. 1992). It should be emphasized that the region around the sites where rearrangements took place, such as deletion/ insertions with multiple nucleotides, translocation, and truncation, are highly AT-rich.

On the other hand, numerous indels in intergenic spacer regions and length variations in protein coding regions result in the size differences in diploid Triticeae species. The rpl2 (in IR regions), petB, petD, rpll6, and rps 12 genes retained their intron in $P$. libanotica, while some of them were absent in the Triticum/Aegilops complex. These intron losses are not unique in diploid Triticeae species. It have been demonstrated that the rpoCl intron is absent in some Poaceae lineages (Katayama and Ogihara 1993). The rpll6 and rpl2 lost their introns in Geraniales (Weng et al. 2014). In addition, protein coding sequences can be responsible for those gene length changes. Most coding regions length variations occurred at the beginning and the end of the sequences. InfA, ranging from 342 to $294 \mathrm{bp}$ in length in Triticeae species, possessed the maximum gene length in $P$. libanotica. It is absent in the Arabidopsis, Lotus, and Medicago cp genomes and present as a truncated pseudogene in ginseng cp genome (Kim and Lee 2004).

The phylogenetic tree inferred from total cp genomes and protein coding regions showed a similar topology while incongruence still existed in the Triticum/Aegilops complex. The reason may be inferred as the whole cp genome possessed more variation sites than coding regions. Parks et al. (2009) illustrated that the noncoding sequences should be especially considered when inferring phylogenies at lower taxonomic levels, resulting from numerous variation sites. Phylogenetic relationship inferred from entire $\mathrm{cp}$ sequences in this study was consistent with two single-copy nuclear genes (Acc1 and $P g k 1$ ) (Fan et al. 2013) as well as the cp genomes from wheat, barley, rye, and their relatives (Middleton et al. 2014).

Evolution of cp genes in Triticeae: Erixon and Oxelman (2008) indicated that positive selection of the $\operatorname{clpP}$ gene in various plant lineages correlates with repeated duplication of the $c l p P$ gene and surrounding regions, repetitive amino acid sequences, and increasing synonymous substitution rates. The $\operatorname{clp} P$ gene in Triticeae contains no repeat but display numerous indels on surrounding regions, which may result in adaptive evolution. However, clpP and rpll6 show a purified pressure in Asteraceae family and Chinese Apiaceae 
subfamily, respectively (Curci et al. 2015). Although a majority of those genes are conserved and essential for cell survival and plant development in some taxa, some genes were disappeared during long evolution. The $c p l P$ genes are totally absent in algae Euglena, Odontella, and Porphyra (Wakasugi et al. 1997). Millen et al. (2001) revealed that $r p l 22, \mathrm{rps} 16$, and infA genes partly disappered during angiosperm evolution or they were completely lost in Legume species.

In accordance with previous investigations, most of genes located in the LSC or SSC regions displayed a more rapid evolution rate compared to sequences in the IR regions (Perry and Wolfe 2002, Qian et al. 2013, Li et al. 2013b, Weng et al. 2014). Some genes in the IR region, however, have a faster divergence than those genes from the LSC or SSC regions. The rpl 2 gene is the fastest evolving gene in diploid Triticeae species. It showed more variations than several genes in the LSC or SSC regions. Usually, majority of the genes in IR ( $n d h B$, rps 15, rps 19, rpl23, rps7, and rps12) regions show no synonymous rate and/or nonsynonymous rate changes while $r p s$ and $r p l$ genes in single-copy regions possess higher divergence. Therefore, the divergence levels of $\mathrm{cp}$ genes are influenced not only by the location of the genes on the cp genome but also by the functional constraints of the genes.

Li et al. (2015) recently has proposed the use of taxon-specific barcodes for species identification using dedicated $\mathrm{cp}$ DNA regions that have a sufficiently high mutation rate. We confirmed the informative values for rps 16, matK, rpoA, rbcL and $n d h F$ regions previously adopted for the phylogenetic evolution of Triticeae species (Mason-Gamer 2002, Sun 2007, Hodge et al. 2010, Dong et al. 2015). Moreover, rpl2, rps 12 (LSC), ccs $A, r p s 8, n d h H$, petD, ndhK, psbM, rps3, rps18, and $n d h A$, exhibiting $\mathrm{K}_{2} \mathrm{P}$ values, which means faster gene divergence, are identified in this work as highly Par. Inf. regions and thus can be considered in future phylogenetic studies, especially $r p l 2$. Although those protein coding genes displayed a fast evolutionary rate and high Par. Inf. characters, genes which possess positive $d_{\mathrm{N}} / d_{\mathrm{S}}$ ratios were also included. As a result, a gene used for phylogeny analysis should have fast evolutionary rate, high Par. Inf. characters and negative $\mathrm{d}_{\mathrm{N}} / \mathrm{d}_{\mathrm{S}}$ ratio. Meanwhile, those informative genes selected for the phylogeny of Triticeae might not adapt to other higher taxonomic level plants. For example, clpP gene which is influenced by positive selective pressure in Triticeae, but it was seemed to be a promising gene which could be utilized to phylogeny analyses in the globe artichoke cp genome with a fairly high Par. Inf. ratio (Curci et al. 2015).

Utility of complete cp genome and cpSSRs in Triticeae phylogenetic analysis: The CpDNA can be used as an effective tool of genealogic studies in Triticeae species (Liu et al. 2006, Zhang et al. 2009, Hodge et al. 2010, Dong et al. 2015). However, a robust phylogenetic analysis requires a substantial sequence length. Nextgeneration sequencing technologies, which have been developed in recent years, enable the determination of the complete nucleotide sequence of both $\mathrm{cp}$ and mitochondrial DNAs of many higher plants, including wheat and its relatives. Entire cp genomes from the Triticum/Aegilops complex species demonstrated that the cp genomes of polyploid wheats were inherited from Speltoides. The Sitopsis lineage was not the source of the B or $\mathrm{G}$ genomes (Gornicki et al. 2014). The whole cp sequences of Ae. tauschii and Ae. cylindrica were used for shedding light on the D genome evolution (Gogniashvili et al. 2016). Additionally, whole cp genomes provided a more detailed insight into the evolution of the Triticum tribe and its relative diploid species than nuclear genes due to $\mathrm{cp}$ divergence date might be closer to the time of species separation (Gornicki et al. 2014, Middleton et al. 2014).

The CpSSRs often display high variation within the same species (Echt et al. 1998) and are considered as valuable markers for population genetics and phylogenetic analyses (Timme et al. 2007, MelottoPassarin et al. 2011). In the $P$. libanotica cp genome, rpoC2 displayed the highest number of repeats with six SSRs, $n d h F$, and $y c f 3$ with five SSRs independently. The rpoC2 and $n d h F$ coding genes have been successfully used in Poaceae genetic relationships (Igloi et al. 1990, Redinbaugh et al. 2000). It is possible that the highly variable $y c f 3$ coding regions are also suitable for phylogenetic analyses or DNA barcoding at low taxonomic levels.

\section{References}

Ayliffe, M.A., Scott, N.S., Timmis, J.N.: Analysis of plastid DNA-like sequences within the nuclear genomes of higher plants. - Mol. Biol. Evol. 15: 738-745, 1998.

Bankevich, A., Nurk, S., Antipov, D., Gurevich, A.A., Dvorkin, M., Kulikov, A.S., Lesin, V.M., Nikolenko, S.I., Pham, S., Prjibelski, A.D., Pyshkin, A.V., Sirotkin, A.V., Vyahhi, N., Tesler, G.,Alekseyev, M.A., Pevzner, P.A.: SPAdes: a new genome assembly algorithm and its applications to singlecell sequencing. - J. comp. Biol. 19: 455-477, 2012.
Curci, P.L., De Paola, D., Danzi, D., Vendramin, G.G., Sonnante, G.: Complete chloroplast genome of the multifunctional crop globe artichoke and comparison with other Asteraceae. - PLoS ONE 10: e120589, 2015.

Dong, Z.Z., Fan, X., Sha, L.N., Wang, Y., Zeng, J., Kang, H.Y., Zhang, H.Q., Wang, X.L., Zhang, L., Ding, C.B., Yang, R.W., Zhou, Y.H.: Phylogeny and differentiation of the St genome in Elymus L. sensu lato (Triticeae; Poaceae) based on one nuclear DNA and two chloroplast genes. - BMC 
Plant Biol. 15: 179-193, 2015.

Echt, C.S., DeVerno, L., Anzidei, M., Vendramin, G.: Chloroplast microsatellites reveal population genetic diversity in red pine, Pinus resinosa Ait. - Mol. Ecol. 7: 307-316, 1998.

Erixon, P., Oxelman, B.: Whole-gene positive selection, elevated synonymous substitution rates, duplication, and indel evolution of the chloroplast $c l p P 1$ gene. - PLoS ONE 3: e1386, 2008

Fan, X., Sha, L.N., Wang, X.L., Zhang, H.Q., Kang, H.Y., Wang, Y., Zhou, Y.H.: Phylogeny and molecular evolution of the $\mathrm{Accl}$ gene within the $\mathrm{StH}$ genome species in Triticeae (Poaceae). - Gene 529: 57-64, 2013.

Frazer, K.A., Pachter, L., Poliakov, A., Rubin, E.M., Dubchak, I.: VISTA: computational tools for comparative genomics. Nucl. Acids Res. 32 (Suppl.): W273-W279, 2004.

Gogniashvili, M., Jinjikhadze, T., Maisaia, I., Akhalkatsi, M., Kotorashvili, A., Kotaria, N., Beridze, T., Dudnikov, A.J.: Complete chloroplast genomes of Aegilops tauschii Coss. and Ae. cylindrica Host sheds light on plasmon D evolution. - Curr. Genet. 62: 791-798, 2016.

Gornicki, P., Zhu, H.L., Wang, J.W., Challa, G.S., Zhang, Z.Z., Gill, B.S., Li, W.L.: The chloroplast view of the evolution of polyploid wheat. - New Phytol. 204: 704-714, 2014.

Guisinger, M.M., Kuehl, J.V., Boore, J.L., Jansen, R.K.: Extreme reconfiguration of plastid genomes in the angiosperm family Geraniaceae: rearrangements, repeats, and codon usage. - Mol. Biol. Evol. 28: 583-600, 2011.

Hodge, C.D., Wang, H., Sun, G.L.: Phylogenetic analysis of the maternal genome of tetraploid StStYY Elymus (Triticeae: Poaceae) species and the monogenomic Triticeae based on rps16 sequence data. - Plant Sci. 178: 463-468, 2010.

Huotari, T., Korpelainen, H.: Complete chloroplast genome sequence of Elodea canadensis and comparative analyses with other monocot plastid genomes. - Gene 508: 96-105, 2012.

Igloi, G.L., Meinke, A., Döry, I., Kössel, H.: Nucleotide sequence of the maize chloroplast rpo $\mathrm{B} / \mathrm{Cl} / \mathrm{C} 2$ operon: comparison between the derived protein primary structures from various organisms with respect to functional domains. - Mol. gen. Genet. 221: 379-394, 1990.

Jensen, K.B.: Cytology, fertility, and origin of Elymus abolinii (Drob.) Tzvelev and its F1 hybrids with Pseudoroegneria spicata, E. lanceolatus, E. dentatus ssp. ugamicus, and E. drobovii (Poaceae: Triticeae). - Genome 32: 468-474, 1989.

Katayama, H., Ogihara, Y.: Structural alterations of the chloroplast genome found in grasses are not common in monocots. - Curr. Genet. 23: 160-165, 1993.

Kearse, M., Moir, R., Wilson, A., Stones-Havas, S., Cheung, M., Sturrock, S., Buxton, S., Cooper, A., Markowitz, S., Duran, C., Thierer, T., Ashton, B., Meintjes, P., Drummond, A.: Geneious basic: an integrated and extendable desktop software platform for the organization and analysis of sequence data. - Bioinformatics 28: 1647-1649, 2012.

Kim, K.J., Lee, H.L.: Complete chloroplast genome sequences from Korean ginseng (Panax schinseng Nees) and comparative analysis of sequence evolution among 17 vascular plants. -DNA Res. 11: 247-261, 2004.

Lee, H.L., Jansen, R.K., Chumley, T.W., Kim, K.J.: Gene relocations within chloroplast genomes of Jasminum and Menodora (Oleaceae) are due to multiple, overlapping inversions. - Mol. Biol. Evol. 24: 1161-1180, 2007.

Li, J.L., Wang, S., Jing, Y., Wang, L., Zhou, S.L.: A modified
CTAB protocol for plant DNA extraction. - Chin. Bull. Bot 48: 72-78, 2013a.

Li, Q, Wan, J.M.: SSRHunter: development of a local searching software for SSR sites. - Hereditas 27: 808-810, 2005.

Li, R., Ma, P.F., Wen, J., Yi, T.S.: Complete sequencing of five Araliaceae chloroplast genomes and the phylogenetic implications. - PLoS ONE 8: e78568, 2013b.

Li, X.W., Yang, Y., Henry, R.J., Rossetto, M., Wang, Y.T., Chen, S.L.: Plant DNA barcoding: from gene to genome. - Biol. Rev. 90: 157-166, 2015.

Liu, Q.L., Ge, S., Tang, H.B., Zhang, X.L., Zhu, G.F., Lu, B.R.: Phylogenetic relationships in Elymus (Poaceae: Triticeae) based on the nuclear ribosomal internal transcribed spacer and chloroplast trnL-F sequences. - New Phytol. 170: 411420, 2006

Lu, B.R.: The genus Elymus L. In: Proceedings of the $2^{\text {nd }}$ International Triticeae Symposium. Pp. 219-233. Logan 1994.

Luo, R.B., Liu, B.H., Xie, Y.L., Li, Z.Y., Huang, W.H., Yuan, J.Y., He, G.Z., Chen, Y.X., Pan, Q., Liu, Y.J., Tang, J.B., Wu, G.X., Zhang, H., Shi, Y.J., Liu, Y., Yu, C., Wang, B., Lu, Y., Han, C.L., Cheung, D.W., Yiu, S.M., Peng, S.L., Zhu, X.Q., Liu, G.M., Liao, X.K., Li, Y.R., Yang, H.M., Wang, J., Lam, T.W., Wang, J.: SOAPdenovo2: an empirically improved memory-efficient short-read de novo assembler. - GigaScience 1: 18, 2012.

Ma, P.F., Zhang, Y.X., Zeng, C.X., Guo, Z.H., Li, D.Z.: Chloroplast phylogenomic analyses resolve deep-level relationships of an intractable bamboo tribe Arundinarieae (Poaceae). - Syst. Biol. 63: 933-950, 2014.

Mariotti, R., Cultrera, N.G., Díez, C.M., Baldoni, L., Rubini, A.: Identification of new polymorphic regions and differentiation of cultivated olives (Olea europaea L.) through plastome sequence comparison. - BMC Plant Biol. 10: $211-223,2010$.

Mason-Gamer, R.J., Orme, N.L., Anderson, C.M.: Phylogenetic analysis of North American Elymus and the monogenomic Triticeae (Poaceae) using three chloroplast DNA data sets. Genome 45: 991-1002, 2002.

Matsuo, M., Ito, Y., Yamauchi, R., Obokata, J.: The rice nuclear genome continuously integrates, shuffles, and eliminates the chloroplast genome to cause chloroplast-nuclear DNA flux. - Plant Cell. 17: 665-675, 2005.

Melotto-Passarin, D., Tambarussi, E., Dressano, K., De Martin, V., Carrer, H.: Characterization of chloroplast DNA microsatellites from Saccharum spp and related species. Genet. mol. Res. 10: 2024-2033, 2011.

Middleton, C.P., Senerchia, N., Stein, N., Akhunov, E.D., Keller, B., Wicker, T., Kilian, B.: Sequencing of chloroplast genomes from wheat, barley, rye and their relatives provides a detailed insight into the evolution of the Triticeae tribe. PLoS ONE 9: e85761, 2014

Millen, R.S., Olmstead, R.G., Adams, K.L., Palmer, J.D., Lao, N.T., Heggie, L., Kavanagh, T.A., Hibberd, J.M., Gray, J.C., Morden, C.W., Calieg, P.J., Jermiinh, L.S, Wolfe, K.H.: Many parallel losses of infA from chloroplast DNA during angiosperm evolution with multiple independent transfers to the nucleus. - Plant Cell 13: 645-658, 2001.

Neuhaus, H., Emes, M.: Nonphotosynthetic metabolism in plastids. - Annu. Rev. Plant Biol. 51: 111-140, 2000.

Ogihara, Y., Terachi, T., Sasakuma, T.: Structural analysis of length mutations in a hot-spot region of wheat chloroplast DNAs. - Curr. Genet. 22: 251-258, 1992. 
Palmer, J.D.: Plastid chromosomes: structure and evolution. Mol. Biol. Plastids 7: 5-53, 1991.

Parks, M., Cronn, R., Liston, A.: Increasing phylogenetic resolution at low taxonomic levels using massively parallel sequencing of chloroplast genomes. - BMC Biol. 7: 84-100, 2009.

Perry, A.S., Wolfe, K.H.: Nucleotide substitution rates in legume chloroplast DNA depend on the presence of the inverted repeat. - J. mol. Evol. 55: 501-508, 2002.

Posada, D., Crandall, K.A.: Modeltest: testing the model of DNA substitution. - Bioinformatics 14: 817-818, 1998.

Provan, J., Powell, W., Hollingsworth, P.M.: Chloroplast microsatellites: new tools for studies in plant ecology and evolution. - Trends Ecol. Evol. 16: 142-147, 2001.

Qian, J., Song, J.Y., Gao, H.H., Zhu, Y.J., Xu, J., Pang, X.H., Yao, H., Sun, C., Li, X.E., Li, C.Y., Liu, J.Y., Xu, H.B., Chen, S.L.: The complete chloroplast genome sequence of the medicinal plant Salvia miltiorrhiza. - PloS ONE 8: e57607, 2013.

Redinbaugh, M.G., Jones, T.A., Zhang, Y.: Ubiquity of the St chloroplast genome in St-containing Triticeae polyploids. Genome 43: 846-852, 2000.

Rodríguez-Ezpeleta, N., Brinkmann, H., Burey, S.C., Roure, B., Burger, G., Löffelhardt, W., Bohnert, H.J., Philippe, H., Lang, B.F.: Monophyly of primary photosynthetic eukaryotes: green plants, red algae, and glaucophytes. Curr. Biol. 15: 1325-1330, 2005.

Rose, O., Falush, D.: A threshold size for microsatellite expansion. - Mol. Biol. Evol. 15: 613-615, 1998.

Rozas, J., Sánchez-DelBarrio, J.C., Messeguer, X., Rozas, R.: DnaSP, DNA polymorphism analyses by the coalescent and other methods. - Bioinformatics 19: 2496-2497, 2003.

Schaack, S., Gilbert, C., Feschotte, C.: Promiscuous DNA: horizontal transfer of transposable elements and why it matters for eukaryotic evolution. - Trends Ecol. Evol. 25: 537-546, 2010.

Sha, L.N., Fan, X., Yang, R.W., Kang, H.Y., Ding, C.B., Zhang, L., Zheng, Y.L., Zhou, Y.H.: Phylogenetic relationships between Hystrix and its closely related genera (Triticeae; Poaceae) based on nuclear Acc1, DMC1 and chloroplast trnL-F sequences. - Mol. Phylogenet. Evol. 54: 327-335, 2010.

Sun, G.: Genetic diversity of $r b c L$ gene in Elymus trachycaulus complex and their phylogenetic relationships to several
Triticeae species. - Genet. Resour. Crop Evol. 54: 17371746, 2007.

Tamura, K., Stecher, G., Peterson, D., Filipski, A., Kumar, S.: MEGA6: molecular evolutionary genetics analysis version 6.0. - Mol. Biol. Evol. 30: 2725-2729, 2013.

Timme, R.E., Kuehl, J.V., Boore, J.L., Jansen, R.K.: A comparative analysis of the Lactuca and Helianthus (Asteraceae) plastid genomes: identification of divergent regions and categorization of shared repeats. - Amer. J. .Bot. 94: 302-312, 2007

Timmis, J.N., Ayliffe, M.A., Huang, C.Y., Martin, W.: Endosymbiotic gene transfer: organelle genomes forge eukaryotic chromosomes. - Natur. Rev. Genet. 5: 123-135, 2004.

Torabinejad, J., Mueller, R.: Genome constitution of the Australian hexaploid grass Elymus scabrus (Poaceae: Triticeae). - Genome 36: 147-151, 1993.

Wakasugi, T., Nagai, T., Kapoor, M., Sugita, M., Ito, M., Ito, S., Tsudzuki, J., Nakashima, K., Tsudzuki, T., Suzuki, Y., Hamada, A., Ohta, T., Inamura, A., Yoshinaga, K., Sugiura, M.: Complete nucleotide sequence of the chloroplast genome from the green alga Chlorella vulgaris: the existence of genes possibly involved in chloroplast division. - Proc. Nat. Acad. Sci. USA 94: 5967-5972, 1997.

Weng, M.L., Ruhlman, T.A., Gibby, M., Jansen, R.K.: Phylogeny, rate variation, and genome size evolution of Pelargonium (Geraniaceae). - Mol. Phylogenet. Evol. 64: 654-670, 2012.

Wicke, S., Schneeweiss, G.M., Müller, K.F., Quandt, D.: The evolution of the plastid chromosome in land plants: gene content, gene order, gene function. - Plant mol. Biol. 76: 273-297, 2011.

Wyman, S.K., Jansen, R.K., Boore, J.L.: Automatic annotation of organellar genomes with DOGMA. - Bioinformatics. 20: 3252-3255, 2004

Yang, Z.H., Bielawski, J.P.: Statistical methods for detecting molecular adaptation. - Trends Ecol. Evol. 15: 496-503, 2000.

Yen, C., Yang, J.L.: Biosystematics of Triticeae. Vol. 4. - China Agricultural Press, Beijing 2011.

Zhang, C., Fan, X., Yu, H.Q., Zhang, L., Wang, X.L., Zhou, Y.H.: Different maternal genome donor to Kengyilia species inferred from chloroplast trnL-F sequences. - Biol. Plant. 53: 759-763, 2009 